\title{
COMMENT
}

Check for updates

\section{Combating physical inactivity during the COVID-19 pandemic}

\author{
Ana Jéssica Pinto (1) ', David W. Dunstan ${ }^{2,3}$, Neville Owen ${ }^{2,4}$, Eloisa Bonfá ${ }^{5}$ and \\ Bruno Gualano ${ }^{1,6 凶}$
}

Physical inactivity is common during periods of self-isolation, but for patients with rheumatic diseases, there are crucial benefits to be gained from maintaining an active lifestyle throughout the COVID-19 pandemic. Patients should be provided with support to maintain physical activity and avoid prolonged periods of time spent sitting.

COVID-19, a disease caused by the SARS-CoV-2 virus, has been classified as a pandemic by the World Health Organization (WHO). In an effort to slow infection rates, particularly in groups predisposed to high risks of morbidity and mortality, extensive social distancing and isolation policies have been adopted worldwide.

Patients with rheumatic diseases commonly have a higher risk of serious infections than the general population owing to immunosuppression and disease activity, particularly in those with suboptimal control of disease activity ${ }^{1}$. Moreover, some patients are at increased risk of COVID-19 complications as they are older and have concomitant chronic diseases. Therefore, patients are recommended by national health advisory services to continue with their current treatment to prevent disease flares and practice self-isolation to prevent infection during the COVID-19 pandemic.

Physical inactivity (not meeting the physical activity guidelines) and sedentary behaviour (too much sitting) are highly prevalent in patients with rheumatic diseases; previous studies show that $38-72 \%$ are physically inactive, and sedentary time ranges between 8.3-14.0 hours/ day, which is higher than for the general population (31\% and $\sim 7.5$ hours/day, respectively $)^{2}$. Importantly, both of these behaviours are associated with poor disease-related outcomes (such as high disease activity, pain and fatigue) and cardiometabolic risk factors (such as obesity and insulin resistance) in patients with rheumatoid arthritis ${ }^{2}$. Among patients with rheumatic diseases, cardiovascular diseases are the leading cause of morbidity and mortality and are likely to be exacerbated by physical inactivity and prolonged periods of sitting. Children and adolescents with rheumatic diseases are also commonly hypoactive compared with healthy peers owing to disease manifestations and symptoms and to overprotection by parents and health-care professionals ${ }^{3}$. The negative clinical effects of inactivity for paediatric patients include muscle atrophy and weakness, fatigue, obesity, insulin resistance, dyslipidaemia, reduced physical capacity and function and poor quality of life ${ }^{3}$.
The COVID-19 pandemic has created an environment that promotes reduced amounts of habitual physical activity owing to self-isolation and quarantine requirements, reduced opportunities to remain physically active and fear of being infected. Sustained physical inactivity and sedentary behaviour are typically associated with poor physical and mental health and increased disease-specific and all-cause mortality risk $^{4}$. Even brief periods of exposure to these behaviours can be deleterious; for example, a 2 -week reduction in daily steps from $\sim 10,000$ to $\sim 1,500$ steps lead to impaired insulin sensitivity and lipid metabolism, increased visceral fat and decreased fat-free mass and cardiovascular fitness in healthy adults ${ }^{5}$. Interestingly, a bout of moderate-intensity exercise does not counteract the detrimental effects of 4 days of inactivity, suggesting that individuals can become 'resistant' to well-known exercise-induced metabolic adaptations ${ }^{6}$.

In the past, bed rest was extensively used as part of the treatment for rheumatic diseases such as rheumatoid arthritis and myositis to avoid disease exacerbation and/or joint destruction. However, compelling evidence shows that disuse actually leads to joint destruction, muscle weakness and atrophy, causing reduced physical function $^{2,3}$. Such evidence has led to the contemporary abrogation of the 'bed rest paradigm' for rheumatic diseases and has guided new approaches to clinical practice that emphasize physical activity as an important part of therapy to improve patients' symptoms ${ }^{7}$. Given that physical inactivity and sedentary behaviour will potentially increase as a function of social distancing measures during the COVID-19 pandemic, patients with rheumatic diseases who are already hypoactive might be at particular risk of worsened disease activity and symptoms, general comorbidities and poor quality of life (FIG. 1).

Exercise (structured physical activity) is advocated by EULAR as an integral part of standard care for patients with inflammatory arthritis and osteoarthritis ${ }^{7}$. Moreover, exercise improves disease symptoms, cardiovascular 


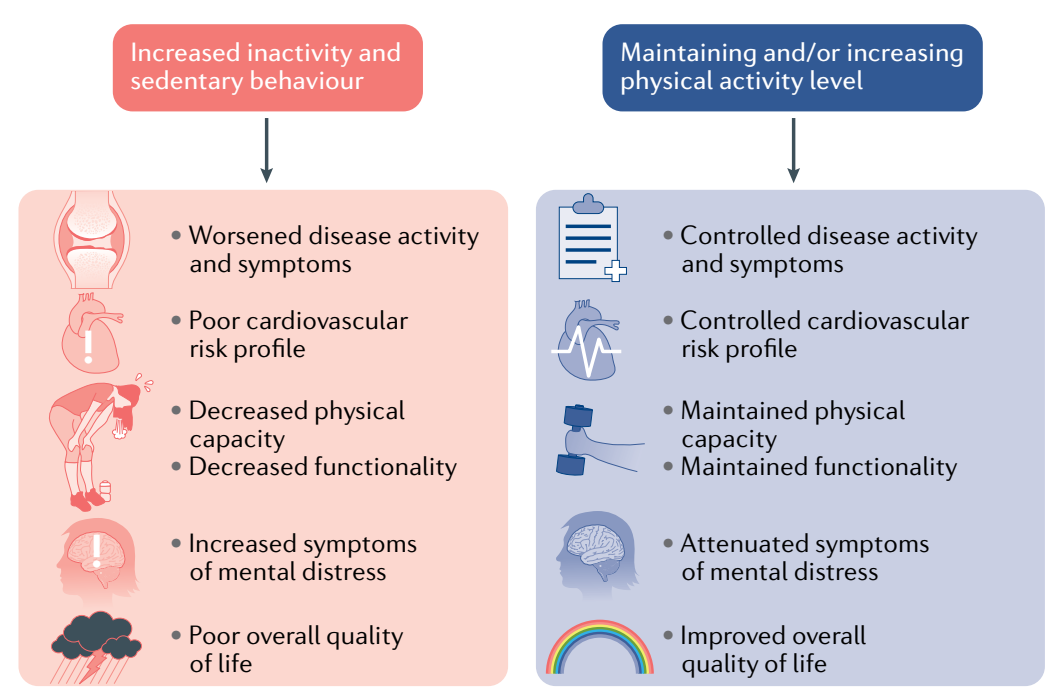

Fig. 1 | The effects of physical inactivity during the COVID-19 pandemic on patients with rheumatic diseases. Imposed physical inactivity and sedentary behaviour (sitting) can be disruptive to disease parameters, cardiovascular risk profile, physical capacity and function and mental health in patients with rheumatic diseases, resulting in poor quality of life, whereas maintaining more optimal physical activity levels can help to ameliorate these detrimental effects.
For patients with particular physical limitations, uncontrolled disease activity and/or who are at high risk of injury when taking unsupervised exercise, home-based exercise programmes designed for the general population might not be ideal from a safety perspective. With these at-risk patients, strategies such as 'move more and sit less during the day' can be promoted as safe and accessible options to at least attenuate the deleterious effects of imposed inactivity during self-isolation. Importantly, simple strategies such as breaking up prolonged sedentary time (such as 2 minutes of walking for every 30 minutes of sitting) could improve some symptoms and cardiometabolic risk factors ${ }^{2}$. As children and adolescents with rheumatic diseases are commonly hypoactive, physical activity should be also recommended to paediatric patients with rheumatic disease ${ }^{3}$.

Patients with rheumatic diseases are already at an increased risk of being hypoactive, which will probably be further aggravated by self-isolation measures imposed to tackle the spread of SARS-CoV-2. This issue raises pressing concerns, as substantial reductions in physical activity can be detrimental to disease outcomes, cardiovascular risk factors, physical capacity and mental health. In light of the systemic benefits of physical activity for patients with rheumatic diseases (FIC. 1), health practitioners are therefore encouraged to be proactive in promoting appropriate physical activity for these patients. and quality of life for patients with other rheumatic diseases $^{8}$. From a safety perspective, acute and long-term exercise did not trigger inflammation in a study involving patients with systemic lupus erythematosus ${ }^{9}$. Of relevance in the context of the COVID-19 pandemic, home-based exercise programmes are feasible and can be effective in promoting health benefits for patients with rheumatic diseases without causing any important adverse events $^{2,3,8}$ (FIG. 1).

Guided by this evidence, we recommend that clinicians and other health-care practitioners are proactive in prescribing physical activities to their patients during the COVID-19 pandemic. Several scientific and medical organizations are promoting messages to keep people active at home during the COVID-19 pandemic, including the $\mathrm{WHO}^{10}$. We highly recommend that associations within the field of rheumatology embrace this idea and help to spread the message that exercise is therapeutic to patients with rheumatic diseases.

What physical activity should be prescribed during self-isolation? In general, patients with rheumatic diseases can follow the current guidelines for the general population. If patients are physically inactive and have no previous experience with exercise programmes, they should engage in less intensive exercise regimes and progress slowly. If patients are already physically active, then they can either maintain their exercise routine, if viable (for example walking), or adapt their activities to be performed at home. A flexible clinical guide for physical activity promotion during the COVID-19 pandemic that can be downloaded as an electronic practitioner resource or shared with patients as a printed pamphlet, electronic document or wall poster is provided as Supplementary Fig. 1. Given patient and practitioner infection concerns about clinical contact, the file can also be adapted for use as a visual resource in telemedicine consultations.
1. Au, K. et al. High disease activity is associated with an increased risk of infection in patients with rheumatoid arthritis. Ann. Rheum. Dis. 70, 785-91 (2011).

2. Pinto, A. J. et al. Physical inactivity and sedentary behavior: overlooked risk factors in autoimmune rheumatic diseases? Autoimmun. Rev. 16, 667-674 (2017). standing up against old paradigms. Nat. Rev. Rheumatol. 13, 368-379 (2017).

4. Booth, F. W. et al. Role of inactivity in chronic diseases: evolutionary insight and pathophysiological mechanisms. Physiol. Rev. 97, 1351-1402 (2017).

5. Krogh-Madsen, R. et al. A 2-wk reduction of ambulatory activity attenuates peripheral insulin sensitivity. J. Appl. Physiol. (1985) 108, 1034-1040 (2010).

6. Akins, J. D. et al. Inactivity induces resistance to the metabolic benefits following acute exercise. J. Appl. Physiol. (1985) 126 1088-1094 (2019).

7. Rausch Osthoff, A. K. et al. 2018 EULAR recommendations for physical activity in people with inflammatory arthritis and osteoarthritis. Ann. Rheum. Dis. 77, 1251-1260 (2018).

8. Perandini, L. A. et al. Exercise as a therapeutic tool to counteract inflammation and clinical symptoms in autoimmune rheumatic diseases. Autoimmun. Rev. 12, 218-224 (2012).

9. Perandini, L. A. et al. Inflammatory cytokine kinetics to single bouts of acute moderate and intense aerobic exercise in women with active and inactive systemic lupus erythematosus. Exerc. Immunol. Rev. 21, 174-185 (2015)

10. World Health Organization. Be active during COVID-19. www.who.int https://www.who.int/news-room/q-a-detail/be-active-during-covid-19 (2020).

\section{Acknowledgements} Amparo à Pesquisa do Estado de São Paulo (2015/26937-4 2018/19418-9; 2017/13552-2 and 2015/03756-4 to A.J.P., B.G. and E.B.), the Conselho Nacional de Pesquisa e Desenvolvimento (305242/2019-9 and 301914/2017-6 to B.G. and E.B.) and the National Health and Medical Research Council of Australia Centre (NHMRC) of Research Excellence (1057608 to D.D. and N.O.). D.D. and N.O. are also supported by the Victorian State Government Operational Infrastructure Support Scheme and by the NHMRC Fellowships Scheme.

\section{Competing interests}

The authors declare no competing interests.

\section{Supplementary information}

Supplementary information is available for this paper at https://doi.org/ 10.1038/s41584-020-0427-z.
3. Gualano, B. et al. Physical activity for paediatric rheumatic diseases:

The work of the authors is supported by grants from the Fundação de 